\title{
H I gas in higher density regions of the IGM
}

\author{
Toru Misawa ${ }^{1}$, David Tytler ${ }^{2,3}$, Masanori Iye ${ }^{4,5}$, Pascal Paschos ${ }^{2}$, \\ Michael Norman ${ }^{2}$, David Kirkman ${ }^{2,3}$, John O'Meara ${ }^{2,3}$, Nao Suzuki ${ }^{2,3}$, \\ and Nobunari Kashikawa ${ }^{4}$ \\ ${ }^{1}$ Department of Astronomy and Astrophysics, Pennsylvania State University, \\ University Park, PA 16802, USA \\ ${ }^{2}$ Center for Astrophysics and Space Sciences, MS 0424, University of California at San Diego, \\ 9500 Gilman Drive, La Jolla, CA 92093-0424, USA \\ ${ }^{3}$ Visiting Astronomer, W. M. Keck Observatory, a Joint Facility of the University of \\ California, the California Institute of Technology, and NASA, USA \\ ${ }^{4}$ National Astronomical Observatory, 2-21-1 Osawa, Mitaka, Tokyo 181-8588, Japan \\ ${ }^{5}$ Department of Astronomical Science, The Graduate University for Advanced Studies, 2-21-1 \\ Osawa, Mitaka, Tokyo 181-8588, Japan
}

\begin{abstract}
Using $\mathrm{H}$ I absorption alone, we attempt to separate $\mathrm{H}$ I absorption lines in quasar spectra into two categories; HDLs (Higher Density Lines) and LDLs (Lower Density Lines), and we discuss the difference in their physical properties. We deblend and fit all $\mathrm{H}$ I lines with Voigt profiles, and make an unbiased sample of $\mathrm{H}$ I lines covering a wide column density range $\left(12<\log N_{H I}<19 \mathrm{~cm}^{-2}\right)$. To reduce the influence of line blending, we simultaneously fit several Lyman series lines. As a result of a two-point correlation analysis, we found that higher column density $\mathrm{H}$ I lines are clustering at $\Delta v<200 \mathrm{~km} \mathrm{~s}^{-1}$, while lower ones at $\Delta v<100 \mathrm{~km} \mathrm{~s}^{-1}$. We define HDLs as $\mathrm{H}$ I lines with $15<\log N_{H I}<19 \mathrm{~cm}^{-2}$ and all $\mathrm{H}$ I lines within $\pm 200 \mathrm{~km} \mathrm{~s}^{-1}$ of a line with $\log N_{H I}>15 \mathrm{~cm}^{-2}$, and LDLs as others with $12<\log N_{H I}<15 \mathrm{~cm}^{-2}$. We found that the HDLs have smaller minimum $b$-values for a given column density than the LDLs. This difference is successfully reproduced by our hydrodynamic simulation. The LDLs seem to be cool or shock-heated diffuse IGM gas, while the HDLs are likely to be cooler dense gas near to galaxies.
\end{abstract}

\section{Introduction}

Quasars have been used as background sources, allowing the study of objects that lie between us and them, most of which produce Ly $\alpha$ absorption lines. Samples of H I absorption lines usually contain (i) $\mathrm{H}$ I lines originating in the intergalactic diffuse gas, and (ii) $\mathrm{H}$ I lines produced near or in intervening galaxies. The former could be weak $\mathrm{H}$ I lines, widely distributed as the Ly $\alpha$ forest (hereafter we call these "LDLs" or "Lower Density Lines"), whereas the latter are likely to be strong H I lines clustered as metal absorption lines (hereafter we call these "HDLs" or "Higher Density Lines"). HDLs are thought to be caused by discrete clouds. On the other hand, LDLs probably arise from photoionised IGMs in the continuous density fields that are broadened by Hubble flow (e.g. Rauch 1998; Kim et al. 2002). However, one of the most serious problems in such an analysis is that the $\operatorname{Ly} \alpha$ absorption lines are so heavily blended with each other that it is difficult to separate and fit them individually.

\section{Data analysis}

We attempt to fit all $\mathrm{H}$ I lines with Voigt profiles, construct an unbiased sample of $\mathrm{H}$ I lines over a wide column density range $\left(12<\log N_{H I}<19\left[\mathrm{~cm}^{-2}\right]\right)$, and investigate their physical parameters, such as column density, Doppler parameter, and clustering 
properties. In order to separate the heavily blended (strong) H I lines and fit them individually with Voigt profiles, we use not only the Ly $\alpha$ line but also higher Lyman series lines, such as $\operatorname{Ly} \beta$ and $\mathrm{Ly} \gamma$, to improve the fitting accuracy (Fig. 1). We apply this fitting method to 40 quasar spectra with resolutions of $8.0 \mathrm{~km} \mathrm{~s}^{-1}$ acquired with the Keck HIRES. We have an unbiased sample of $973 \mathrm{H}$ I lines in 61 systems, where a system means line clustering within $1,000 \mathrm{~km} \mathrm{~s}^{-1}$. As a result of a two-point correlation analysis, we found that higher column density $\mathrm{H}$ I lines with $\log N_{H I} \geqslant 15$ are clustering at $\Delta v<200 \mathrm{~km} \mathrm{~s}^{-1}$, while lower ones are at $\Delta v<100 \mathrm{~km} \mathrm{~s}^{-1}$. We therefore define HDLs as H I lines with $15 \leqslant \log N_{H I}<19\left[\mathrm{~cm}^{-2}\right]$, although the value of $\log N_{H I}=15$ is not strict. These strong $\mathrm{H}$ I lines may also be accompanied by weak $\mathrm{H}$ I lines that are physically associated with them. In fact, metal absorption lines sometimes have a core-halo structure; the strongest line is at the centre of the absorption system, whereas the weak lines exist almost symmetrically on both sides of the strong one (e.g. Lu et al. 1996; Prochaska et al. 2001). Therefore we also classify weak H I lines into HDLs, if these are within $\pm 200 \mathrm{~km} \mathrm{~s}^{-1}$ of the strong $\mathrm{H}$ I lines with $\log N_{H I} \geqslant 15$. LDLs are defined as others with $12<\log N_{H I}<15$. All $\mathrm{H}$ I lines were classified into 306 HDLs and 667 LDLs. We found the most remarkable difference between HDLs and LDLs in the plot of column density vs. Doppler parameter. When we ignore $\mathrm{H}$ I lines with $b<15 \mathrm{~km} \mathrm{~s}^{-1}$, we found a positive correlation between the minimum Doppler parameter $b_{\text {min }}$ and column density, not only for LDLs, approximately fitted by

$$
b_{\text {min }}=4.0 \times \log \left[\frac{N_{H I}\left(\mathrm{~cm}^{-2}\right)}{10^{12.5}}\right]+14.0 \mathrm{~km} \mathrm{~s}^{-1}
$$

at $12.5<\log N_{H I}<15.0$ as seen in the past papers (e.g. Kirkman \& Tytler 1997); but also for HDLs fitted by

$$
b_{\text {min }}=1.3 \times \log \left[\frac{N_{H I}\left(\mathrm{~cm}^{-2}\right)}{10^{12.5}}\right]+10.5 \mathrm{~km} \mathrm{~s}^{-1}
$$

at $12.5<\log N_{H I}<19.0$. The HDLs have smaller minimum $b$-values for a given column density than the LDLs, which suggests that H I absorbers are not produced by a single phase (or a single population). This difference is successfully reproduced by our three-dimensional hydrodynamic simulation in a CDM-dominated Universe $\left(\Omega_{b}=\right.$ $0.04, \Omega_{m}=0.30, \Omega_{\Lambda}=0.70, H_{0}=70 \mathrm{~km} \mathrm{~s}^{-1} \mathrm{Mpc}^{-1}, \sigma_{8}=0.73$, and power spectrum slope of $n=1$ ) (Misawa et al. 2004).

\section{Discussion}

The LDLs seem to be cool or shock-heated diffuse IGM gas, while the HDLs are likely to be cooler dense gas near to galaxies. When we think about $\mathrm{H}$ I clouds in the cold dense phase, the effect of radiative cooling must be taken into consideration. Such dense clouds with $\log N_{H I} \geqslant 17$ (i.e. LLSs and DLA systems) can shield themselves against the background UV flux (i.e. the shielding effect). A similar effect may happen in the low column density clouds if they are located near the high column density clouds (i.e. the shadowing effect). These effects are important at least at $z \geqslant 6$ (Nakamoto, Umemura \& Susa 2001), although they have not yet been evaluated quantitatively at lower redshift because of the more complex situation after the formation of the first stars and quasars. Zhang, Anninos \& Norman (1995) also pointed out the importance of the shielding effect, though they did not include the effect in their simulation, and nor did we here. If plenty of $\mathrm{H}$ I gas is preserved, the cooling of $\mathrm{H}$ I gas becomes very effective in regions in photoionisation equilibrium ( $\mathrm{H}$ I line cooling is the most effective cooling 


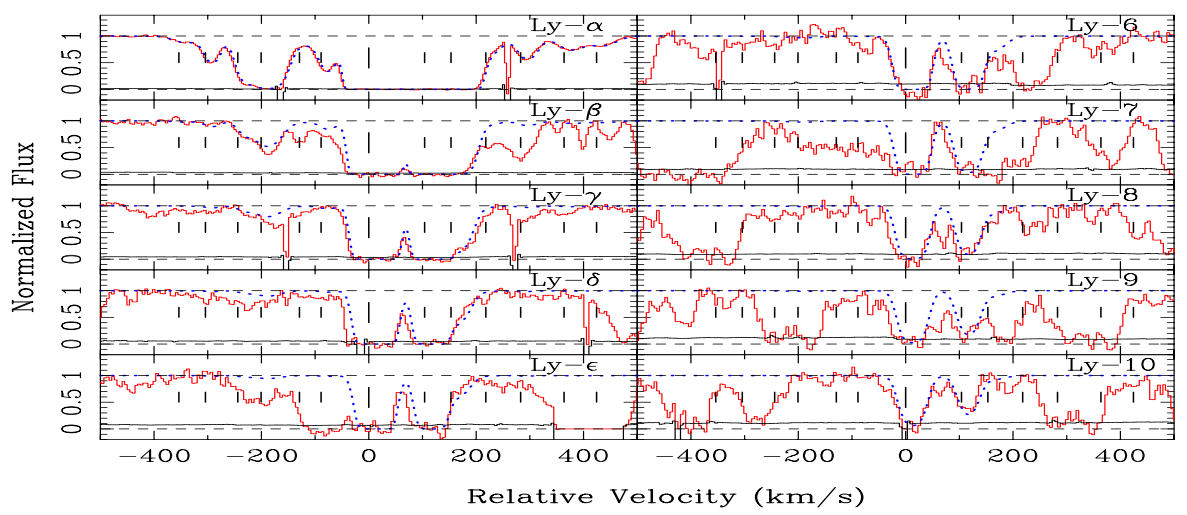

Figure 1. Observed (solid) and modelled (dashed) line profiles for 10 Lyman series transitions of the $\mathrm{H}$ I lines at $z_{a b s}=3.321$ in the spectrum of Q0014+8118. Tick marks denote the positions of $\mathrm{H}$ I absorption lines, and the large one at the centre is the position of the strongest component.

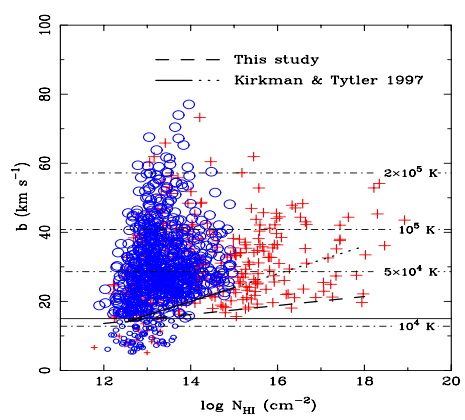

Figure 2. Doppler parameter vs. column density. Open circles and crosses are LDLs and HDLs, respectively. The solid (and dotted) line and the dashed line are the relations between $\log N_{H I}$ and $b_{\text {min }}$ for LDLs and HDLs. The pure thermal Doppler parameters corresponding to the temperatures, $T=10^{4}, 5 \times 10^{4}, 10^{5}$, and $2 \times 10^{5} \mathrm{~K}$, are also shown with the thin dot-dash horizontal lines.

factor at $T \sim 10^{4} \mathrm{~K}$ ), which could lower the temperature, and decrease the value of the minimum Doppler parameter. We speculate that both of these effects play important roles in accounting for the difference of the $\mathrm{H}$ I distributions in the $\log N-b$ plane between LDLs and HDLs.

\section{Acknowledgements}

This work was supported in part by NASA grant NAG5-13113.

\section{References}

Kim, T.-S., Carswell, R. F., Cristiani, S., D’Odorico, S., Giallongo, E., 2002, MNRAS, 335, 555 Kirkman, D., Tytler, D., 1997, ApJ, 484, 672

Lu, L., Sargent, W. L. W., Barlow, T. A., Churchill, C. W., Vogt, S. S., 1996, ApJS, 107, 475

Misawa, T., Tytler, D., Iye, M., Paschos, P., Norman, M., Kirkman, D., O'Meara, J., Suzuki, N., Kashikawa, N., 2004, AJ, 128, 2954

Nakamoto, T., Umemura, M., Susa, H., 2001, MNRAS, 321, 593

Prochaska, J. X., Wolfe, A. M., Tytler, D., Burles, S., Cooke, J., Gawiser, E., Kirkman, D., O'Meara, J. M., Storrie-Lombardi, L., 2001, ApJS, 137, 21

Rauch, M., 1998, ARA\&A, 36, 267

Zhang, Y., Anninos, P., Norman, M. L., 1995, ApJ, 453, L57 